\title{
Factors for compliance with infection control practices in home healthcare: findings from a survey of nurses' knowledge and attitudes toward infection control
}

\author{
David Russell, PhDa,b, ${ }^{\text {, }}$, Dawn W. Dowding, PhD, RN, FAAN ${ }^{c}$, Margaret V. McDonald, MSW ${ }^{b}$, \\ Victoria Adams, BSN, MSN, FNP-BC ${ }^{d}$, Robert J. Rosati, PhD ${ }^{e}$, Elaine L. Larson, PhD, RN, \\ FAAN, CIC $^{d}$, and Jingjing Shang, PhD, RN ${ }^{d}$ \\ aDepartment of Sociology, Appalachian State University, Boone, NC \\ ${ }^{b}$ Center for Home Care Policy \& Research, Visiting Nurse Service of New York, New York, NY \\ 'Division of Nursing, Midwifery and Social Work, School of Health Sciences, University of \\ Manchester, Manchester, United Kingdom \\ ${ }^{d}$ Columbia University School of Nursing, New York, NY \\ eVisiting Nurse Association Health Group, Holmdel, NJ
}

\section{Abstract}

Background-Infection is a leading cause of hospitalization among home healthcare patients. Nurses play an important role in reducing infection among home healthcare patients by complying with infection control procedures. However, few studies have examined the compliance of home healthcare nurses with infection control practices or the range of sociocultural and organizational factors that may be associated with compliance.

Methods-This study analyzed survey responses from nurses at 2 large, certified home healthcare agencies $(n=359)$, to explore levels of compliance with infection control practices and identify associated demographic, knowledge, and attitudinal correlates.

Results-Nurses reported a high level of infection control compliance (mean $=0.89$, standard deviation $[\mathrm{SD}]=0.16$ ), correct knowledge (mean $=0.85, \mathrm{SD}=0.09$ ), and favorable attitudes (mean $=0.81, \mathrm{SD}=0.14)$. Multivariate mixed regression analyses revealed significant positive associations of attitudinal scores with reported level of compliance $(P<.001)$. However, knowledge of inflection control practices was not associated with compliance. Older $(P<.05)$ and non-Hispanic black $(P<.001)$ nurses reported higher compliance with infection control practices than younger and white non-Hispanic nurses.

Conclusion-These findings suggest that efforts to improve compliance with infection control practices in home healthcare should focus on strategies to alter perceptions about infection risk and other attitudinal factors.

\footnotetext{
*Address correspondence to David Russell, PhD, Department of Sociology, Appalachian State University, ASU Box 32115, 209 Chapell Wilson Hall, 480 Howard Street, Boone, NC 28608-2115. russelldj@ appstate.edu (D. Russell).

Conflicts of interest: The authors declared no potential conflicts of interest with respect to the research, authorship, and/or publication of this article.
} 


\section{Keywords}

Infection; Home healthcare; Nursing; Compliance; Attitudes; Knowledge

Infection is prevalent among the more than 4 million patients in the United States who receive skilled home healthcare (HHC) services each year and is a leading cause of unplanned hospitalization. ${ }^{1,2}$ Estimates based on 2010 national Outcome and Assessment Information Set data have attributed more than $17 \%$ of all unplanned hospitalizations among HHC patients to respiratory, urinary tract, intravenous catheter-related, and wound infections. ${ }^{3}$ Many infections acquired by patients can be spread by healthcare workers. ${ }^{4}$ One study found that $5.9 \%$ of $\mathrm{HHC}$ workers reported that they were diagnosed with an occupationally acquired infection caused by a multidrug-resistant organism (MDRO). ${ }^{5} \mathrm{~A}$ second study of 126 nurses from 4 HHC agencies found that the nurse's bag can serve as a potential reservoir for bacterial organisms-with $83.6 \%$ of bags testing positive for human pathogens, of which $15.9 \%$ were MDROs. ${ }^{6}$ Nurses play an especially important role in the spread of infections to patients, because they are responsible for more than half of all HHC visits and perform invasive procedures, including wound treatments and insertions of urinary catheters. ${ }^{1,5}$ The Centers for Medicare and Medicaid Services has recently updated the Conditions of Participation for HHC to place a greater emphasis on infection control (IC) practices, to ensure they are more in line with other providers, such as hospitals and skilled nursing facilities. ${ }^{7}$ Many infections are preventable through compliance with evidence-based IC strategies. ${ }^{8}$ HHC nurses' compliance with standard precautions, including hand hygiene, adherence to aseptic techniques, and use of personal protective equipment (including gloves, gowns, masks, eye protection, and face shields), can be instrumental in preventing the spread of infections among patients and providers. ${ }^{5,9}$ Although researchers have highlighted the importance of standard precautions in HHC, few studies have examined HHC nurses' infection control practices, despite the many challenges that these workers face in what is an autonomous and often unpredictable setting. 5,10

Compliance with IC practices, including hand hygiene and use of personal protective equipment, has been found to vary widely among healthcare workers and is likely influenced by one's knowledge and attitudes about infection risk and behaviors. ${ }^{1-13}$ Much of what is currently known about healthcare-associated infections is based on research conducted in settings other than HHC (eg, hospitals). This research indicates that IC compliance tends to be less than optimal, especially for precautions that are directly observable. ${ }^{14,15}$ Healthcare providers have been found to demonstrate poor compliance with hand hygiene practices despite well-established guidelines for the prevention of healthcare-associated infections. 16-18 Poor compliance with IC practices may stem from knowledge deficits and/or attitudes, including perceived barriers such as lack of time, the sense that protective equipment interferes with work performance, equipment availability, or patient discomfort. ${ }^{15,19,20}$

Figure 1 shows a conceptual diagram based on the ecological model and theory of planned behavior that guided our analysis of compliance with IC practices. ${ }^{21,22}$ The ecological perspective emphasizes the social and organizational context of health behaviors, including sociodemographic factors (eg, age, education, and nursing experience) and training 
characteristics (eg, receipt of IC training and certification). ${ }^{21}$ Furthermore, the theory of planned behavior suggests that compliance with IC practices will be positively influenced by favorable attitudes (eg, that infections pose a serious risk to HHC patients) as well as by the information accuracy of IC knowledge (eg, applicability of standard precautions to different patient groups). ${ }^{23,24}$ This study had the following 2 aims: 1) to describe HHC nurses' levels of IC knowledge, attitudes, and practices; and 2) to examine relationships between HHC nurses' demographic and training characteristics, levels of knowledge, and attitudes toward IC practices, and self-reported IC compliance.

\section{METHODS}

\section{Study design, setting, and survey recruitment procedure}

In this study, data were collected regarding nurses' IC knowledge, attitudes, and practices through an online survey administered at 2 large, not-for-profit, Medicare-certified HHC agencies located in the northeastern United States. In 2016, these 2 agencies collectively employed over 1,000 nursing personnel and served more than 150,000 patients across both urban (Agency 1) and suburban (Agency 2) areas. Eligible survey respondents included registered nurses and licensed practical/vocational nurses who were actively employed by 1 of the 2 surveyed HHC agencies in roles as full-time staff nurse, part-time staff nurse, or team manager. Study procedures were approved by the institutional review boards at Columbia University Medical Center, the Visiting Nurse Service of New York, and the Visiting Nurse Association Health Group. Then, a list of the names and e-mail addresses of all eligible nurses was obtained from each agency's human resources department.

The survey was administered between April 25 and August 20, 2017. E-mail invitations were sent to 1,134 nurses across the 2 agencies. To encourage participation, 3 weekly reminders were sent to nurses during this period. A total of 415 responses were received, resulting in a $36.6 \%$ response rate. The analytic sample included 359 nurses with valid and complete responses for all survey items, including 210 nurses from Agency 1 and 149 nurses from Agency 2. Not included in the analysis were 56 nurses who provided only partial responses to the survey, including 34 nurses (61\% of all nurses with partial responses) who did not give demographic and training information; 14 nurses (25\%) who did not complete the compliance section of the questionnaire; and 8 nurses (14\%) who did not complete questions about knowledge and/or attitudes toward IC practices.

\section{Development and testing of survey instrument}

Survey questionnaire items were developed to assess knowledge, attitudes, and practices regarding IC. Items were reviewed by experts in the field of IC, including 1 nationally recognized IC researcher and 1 employee at Agency 1 who worked to develop the organization's IC policies and procedures. Together, these experts evaluated the face validity of the survey by checking the questionnaire's applicability to HHC nurse practice and ensuring its consistency with established agency policies and procedures.

Knowledge of IC protocols was measured with 7 items informed by and adapted from previously developed IC instruments. ${ }^{25-27}$ Items included 2 statements in which nurses 
indicated their level of agreement by selecting 1 of the following response options: agree, uncertain, or disagree (eg, "Standard precautions do not need to be applied to persons without infections"; correct response was "disagree"). Five additional multiple-choice questions were asked of nurses, from which 21 individual response options were coded as correct or incorrect (eg, "Which of the following sources can harbor harmful germs on hands?"; response options included "Jewelryworn on the hands" [Yes], "Damaged skin" [Yes], "Artificial fingernails" [Yes], "Birthmarks" [No]). In all, 23 knowledge responses were coded as correct or incorrect. These individual items were then used to calculate the proportion of correct responses across all items. This proportional score ranges from 0 to 1 , where 1 reflects correct knowledge on all items and 0 indicates incorrect knowledge on all items.

Attitudes regarding IC protocols were assessed with 17 items that captured the level of agreement or disagreement with statements about the seriousness of infections within HHC settings and the risks they pose to healthcare workers, awareness and education of IC procedures, and resources provided by the employing agency (eg, "Infections are a serious problem in home care"; response options included "strongly agree" [appropriate response], "agree" [appropriate response], "neither agree nor disagree," "disagree," and "strongly disagree"). Responses were coded as appropriate or inappropriate and then used to calculate the proportion of appropriate attitudes across all items. This proportional score ranges from 0 to 1 , where 1 reflects that appropriate attitudes were indicated for all statements and 0 indicates inappropriate attitudes for all items.

Compliance with IC practices was assessed with 8 items adapted from previously developed instruments ${ }^{27-29}$ and based on the HHC agency's IC policies. These items assessed compliance with hand hygiene practices, use of appropriate supplies when in contact with bodily fluids, and proper disposal of contaminated materials (eg, "I dispose of needles in a sharps container"; response options included "always" [appropriate response], "often" [appropriate response], "sometimes" [inappropriate response], "rarely" [inappropriate response], and "never" [inappropriate response]). Responses were coded as compliant or noncompliant and then used to calculate the proportion of compliant responses across all items. This proportional score ranges from 0 to 1 , where 1 reflects self-reported compliance with all practices and 0 indicates noncompliance with all practices.

Several demographic and training characteristics of nurses were measured on the survey and examined in our analysis, including age; sex; race/ethnicity (ie, non-Hispanic white, nonHispanic black, Hispanic, other, or mixed race/ethnicity); highest level of educational attainment (ie, associate's degree or some college, bachelor's degree, or graduate degree); number of years in the nursing profession; number of years in HHC nursing; number of years working at one's current HHC agency; position at the HHC agency (ie, fulltime staff nurse, part-time staff nurse, or team manager); last time that the respondent received IC training (ie, less than 6 months ago, more than 6 months ago but less than 1 year, or more than 1 year ago); and whether one had received certification in IC.

Prior to administering the survey to our targeted sample, the survey instrument was piloted with $47 \mathrm{HHC}$ nurses at 2 agencies in Pennsylvania and New York who were not part of the 
analytic sample. Feedback obtained through open-ended questions was used to modify the questionnaire to improve clarity and relevance for the HHC nurse population.

\section{Data analysis}

Univariate statistics, including means, percentages, and proportions, were used to summarize nurse demographics, IC training characteristics, and responses to IC knowledge, attitudes, and self-reported compliance items. Chi-square tests and t-tests were employed to assess for statistically significant differences $(P<.05)$ in nurse characteristics between the 2 surveyed agencies. A multivariate linear mixed regression model was estimated to examine variation in compliance with IC practices (dependent variable). Independent variables included nurse sociodemographics, IC training characteristics, and proportional scores for IC knowledge and attitudes. Agency was specified as an intercept-level random effect, whereas all other independent variables were specified as fixed effects. A $P$ value of .05 was used as a threshold for determining statistical significance in the regression results.

\section{RESULTS}

\section{Characteristics of nurse survey respondents}

The characteristics of nurse survey respondents are summarized in Table 1. The average age of participants was 50 years (standard deviation $[\mathrm{SD}]=10.5$ ). Most participants were women $(91.6 \%)$, white non-Hispanic (42.9\%) or black non-Hispanic (24.0\%), and had a bachelor's (51.8\%) or graduate (19.8\%) degree. Nurses had an average of more than 20 years of nursing experience (mean $=21.9 ; \mathrm{SD}=11.9)$, approximately 14 years of HHC experience (mean $=13.5 ; \mathrm{SD}=9.3$ ), and nearly 12 years of experience at their current agency $($ mean $=11.5 ; \mathrm{SD}=9.1)$. Most respondents were full-time staff nurses $(64.9 \%)$, followed by part-time staff nurses $(21.4 \%)$ and team managers (13.6\%). The vast majority of nurses reported having received IC training in the previous year, with more than a third $(39.3 \%)$ reporting having received IC training in the previous 6 months. However, less than a fifth $(18.1 \%)$ of nurses had formal IC certification. The surveyed agencies differed with respect to the race/ethnic and educational composition of nursing staff, with Agency 1 having significantly more minority group representation and a larger percentage of nurses with graduate degrees. Nurses surveyed at Agency 1 also tended to have significantly longer tenure at their organization compared to those surveyed from Agency 2 (Agency 1 mean = 13.7 years vs Agency 2 mean $=8.3$ years; $P<.001$ ). The demographic characteristics of respondents were compared to the entire population of nurses at each surveyed agency. These analyses revealed that survey respondents did not differ from the agency nurse populations on measures of sex, age, or agency tenure. However, we did find that black nurses were under-represented in our survey sample relative to the total population of nurses at each agency (Agency 1: $26.7 \%$ of sample vs $41.2 \%$ of population; Agency 2: $20.1 \%$ of sample vs $25.8 \%$ of population). Furthermore, nurses with graduate degrees were overrepresented in our sample relative to the total population of nurses at each agency (Agency 1: $26.7 \%$ of sample vs $11.5 \%$ of population; Agency 2: $10.1 \%$ of sample vs $3.2 \%$ of population). 
Proportional scores for each of the 3 IC measures (ie, knowledge, attitudes, and compliance with IC practices) are also shown. These mean scores ranged from $0.89(\mathrm{SD}=0.16)$ for compliant practices, $0.85(\mathrm{SD}=0.09)$ for correct knowledge selections, and $0.81(\mathrm{SD}=$ 0.14 ) for appropriate attitudinal responses. Nurses surveyed at Agency 1 reported significantly lower compliance scores than nurses from Agency 2 (Agency 1 mean $=0.88$ $[\mathrm{SD}=0.16]$ vs Agency 2 mean $=0.92[\mathrm{SD}=0.15] ; P<.05)$. Proportional scores for IC knowledge and attitudes did not differ significantly between the 2 surveyed agencies.

\section{Knowledge, attitudes, and compliance with IC practices}

Table 2 presents the percentages of correct knowledge responses on each questionnaire item for survey respondents. The vast majority of nurses provided correct responses to items concerning the applicability of standard precautions to people without infections (98.9\%), healthcare workers who have contact with bodily fluids (93.9\%), and their relevance to both patients and healthcare workers (92.5\%). Correct responses were also frequent for items regarding the development of MDROs through antibiotic overuse (97.8\%) and the applicability of contact precautions to patients with MDROs (93.9\%), as well as for items referencing the harboring of microbes on hands through artificial fingernails (96.9\%), jewelry (95.3\%), birthmarks (incorrect response; 93.6\%), and damaged skin (88.0\%). Fewer correct responses were found for items concerning the necessity of masks/goggles for care that is unlikely to cause splashing of fluids (69.6\%); using soap and water to wash hands before eating and drinking $(57.1 \%)$; and for items regarding the nursing bag, including performing hand hygiene after touching the bag $(70.8 \%)$ and the number of necessary bag compartments $(10.3 \%)$. Differences between agencies in the percentage of correct knowledge responses were found for 3 items: nurses surveyed at Agency 1 were less likely to correctly identify appropriate hand-washing techniques (ie, using alcohol-based hand rub in lieu of soap and water) when eating or drinking (51.0\% in Agency 1 vs 65.8\% in Agency $2 ; P<.01)$ or when hands were not visibly soiled $(79.0 \%$ v. $88.6 \% ; P<.05)$. Nurses surveyed at Agency 1were also less likely than nurses from Agency 2 to endorse the statement that standard precautions include recommendations to protect both patients and healthcare workers $(90.0 \%$ v. $96.0 \% ; P<.05)$.

The percentages of appropriate responses to each of the 17 items measuring attitudes toward IC practices are displayed in Table 3. Approximately two-thirds (67.7\%) of nurses surveyed agreed that infections are a serious problem in home care. A higher percentage (96.4\%) agreed with the statement that patients can develop infections from contact with people who visit or live with them and can spread their infection to healthcare workers (81.3\%). Most nurses expressed agreement that hand hygiene protects patients from infection $(98.1 \%)$ and that their agency makes hand hygiene products and masks easily accessible $(95.8 \%$ and $89.4 \%$, respectively). Fewer appropriate responses were given for items concerning the difficulty of communicating with patients when wearing masks (44.3\%), the ease with which agencies allow nurses to stay at home when they are sick (60.4\%), and the perceived safety of influenza vaccinations (69.9\%). Differences in attitudinal responses between surveyed agencies were identified for 1 item: nurses surveyed at Agency 1 were less likely to disagree with the statement that wearing a mask makes it hard to communicate with patients (38.6\% v. $52.3 \% ; P<.01)$. 
Percentages of compliant responses to 8 self-reported IC practices are presented in Table 4. Very high compliance was reported for several practices, including wearing gloves when anticipating exposure to bodily fluid or blood (100.0\%); performing hand hygiene before and after patient care activities (99.4\%); disposal of needles in a sharps container (96.4\%); performing hand hygiene immediately after removing gloves (95.5\%); and properly disposing contaminated materials (91.9\%). Lower compliance was reported for IC practices involving wearing goggles or eye shields when exposed to bodily fluids (69.6\%); wearing a gown if soiling with bodily fluids is likely (78.8\%); and wearing a disposable face mask whenever there is a possibility of splash or splatter (81.9\%). Two significant differences were noted between surveyed agencies in their compliance: nurses surveyed from Agency 1 were less likely to report wearing goggles or eye shields when exposed to bodily fluids (64.8\% in Agency 1 vs $76.5 \%$ in Agency 2; $P<.05$ ) and were less likely to report wearing a disposable face mask whenever there is possibility of splash or splatter $(78.1 \%$ vs $87.2 \% ; P$ $<.05)$.

\section{Multivariate analyses of compliance with IC practices: relationships with nurse demographic characteristics, knowledge, and attitudes toward IC protocols}

Table 5 presents the results from a multivariate linear mixed regression model examining the level of self-reported compliance with IC practices, controlling for nurse demographics, training characteristics, and measures of IC knowledge and attitudes. The results revealed significant associations between IC compliance and nurse demographic characteristics. For instance, older nurses, non-Hispanic black nurses, and nurses with IC certification reported greater compliance with IC practices than younger nurses $(\beta=0.003, P<.05)$, nonHispanic white nurses $(\beta=0.072, P<.001)$, and nurses without IC certification $(\beta=0.047$, $P<.05)$. In addition to these demographic and training characteristics, the model results also indicated that nurses' attitudes regarding IC practices were positively and significantly associated with IC compliance $(\beta=0.236, P<.001)$. Knowledge scores were not significantly associated with compliance scores.

\section{DISCUSSION}

Our study results suggest a high rate of self-reported compliance with most IC practices among nurses surveyed at 2 large $\mathrm{HHC}$ agencies. The percentage of nurses in our sample who reported compliance with IC practices exceeded $90 \%$ for 5 of 8 measured behaviors. The high rates of self-reported compliance observed in our study are consistent with previous work that also indicated high rates of self-reported compliance with IC practices, including those for standard precautions, and strong agreement with statements about the importance of IC procedures. ${ }^{15,30-32}$ However, our findings differ from those of researchers who directly observed nurses' IC practices. Studies using direct observation methods have found poor compliance with hand hygiene practices. ${ }^{33}$ These contradictory findings suggest that nurses may indicate compliance that is higher than what would have been directly observed and more attributable to their intentions or perceptions about IC practices. ${ }^{30,31}$

Findings regarding knowledge of IC practices were mixed. On the one hand, our results suggested adequate knowledge among nurses regarding most features of standard 
precautions, protocols for handling exposure to bodily fluids, and mechanisms for the spread of MDROs. On the other hand, we found a lesser degree of knowledge concerning required features of the nursing bag, hand hygiene practices, and the necessity of masks and goggles for care that is unlikely to cause splashing of fluids. These findings differ somewhat from those of others, who found acceptable knowledge of both hand hygiene practices and standard precautions among first-year nursing students in France. ${ }^{25}$ These differences could be due to the way survey items were worded, variations by country or healthcare setting, or the fact that their sample included recently trained students, whereas we examined practicing nurses, many of whom were educated several decades prior to the time of survey. For instance, our study identified opportunities to improve nurses' knowledge of IC practices related to the nursing bag, which had relatively low knowledge compared to other survey topics. More than a quarter of the nurses we studied failed to identify that hand hygiene should be performed after touching the nursing bag. Furthermore, nearly $90 \%$ of the sample did not recognize that the nursing bag should have at least 3 separate compartments, despite agency policies stating that multi-use items (eg, blood pressure cuffs and stethoscopes) must be stored separately from single-use items (eg, sterile supplies and personal protective equipment), and that hand hygiene supplies must be stored in a separate (ie, third) compartment so that hands can be cleaned before entering other compartments. These gaps in knowledge are notable considering that that the nursing bag could potentially serve as a vector for transporting infectious pathogens between HHC patients. HHC nurses may benefit from additional training on IC practices regarding the nursing bag, including protocols that have been shown to reduce infection risk, such as use of less porous surface materials, use of institutional cleaners containing sodium hypochlorite, and daily cleaning of the outside of the bag. ${ }^{6}$

In addition to gaps in knowledge for some IC precautions, we also observed unfavorable attitudes toward certain infection prevention practices. For instance, only slightly more than two-thirds $(68.5 \%)$ of nurses in our sample indicated agreement that the influenza vaccine is safe, and an even smaller percentage (60.4\%) of nurses thought that it was easy for them to stay at home when they are sick. These findings echo those of a previous study, which found that many HHC staff who refused the influenza vaccination thought that they did not need the vaccination or that it would make them sick. ${ }^{34}$ Given the strong association observed between IC attitudes and compliance, these results suggest that HHC agencies may benefit from offering their staff educational programs on influenza vaccination and employee sick leave policies.

Our findings provide support for the ecological model as well as the theory of planned behavior, which consider both the environmental context of health behaviors and one's own attitudes toward those behaviors. ${ }^{21,35}$ In support of the ecological model, our results indicated the importance of individual sociodemographics (ie, age and race/ethnicity) and agency characteristics (ie, receipt of IC certification) for IC compliance. Echoing previous research informed by the theory of planned behavior, we did not find a statistically significant relationship between level of IC knowledge and self-reported compliance with IC practices. The absence of a significant relationship between these 2 factors suggests that differences between HHC nurses in their IC knowledge are alone insufficient to account for variations in compliance. This finding is consistent with previous research among nurses in 
acute hospitals, which found no significant relationships between nurses' levels of knowledge and self-reported compliance. ${ }^{28}$ Rather, our study revealed a significant positive association between IC attitudes and compliance, indicating that compliance with IC practices among HHC nurses may be driven more by subjectively held information than by the accuracy of one's knowledge. For instance, nurses who perceive a greater risk of infection may be more likely to comply with IC practices than those who perceive a lesser risk of infection. This would be consistent with earlier work that found that attitudes and beliefs about hand washing were strong predictors of compliance rates, leading the researchers to conclude that self-protection may be a major driving force in IC compliance. ${ }^{36,37}$ Further research involving direct observation of nurses' IC practices, and an investigation into the meanings or beliefs that those practices have for nurses, could help to shed light on compliance in the HHC setting. Drawing on lessons learned from research on the theory of planned behavior, efforts to improve compliance with IC practices should move beyond a singular focus on knowledge and share informative messages with healthcare workers that attempt to challenge their existing beliefs (eg, e-mail communications that dispel common misconceptions about influenza vaccination). ${ }^{35}$ This approach has been shown to be effective in promoting hand hygiene. ${ }^{38}$ Furthermore, agencies will need to tailor IC programs that were originally developed in hospital settings for the unique characteristics of and risks associated with HHC. ${ }^{39}$

Strengths of our study included a large sample of nurses from $2 \mathrm{HHC}$ agencies that were diverse in terms of both their demographic and IC training characteristics. Moreover, the survey questionnaire included a broad range of questions measuring IC knowledge, attitudes, and practices that were informed by previous research yet tailored by the research team to be relevant to the HHC setting.

Despite these strengths, our study did have notable limitations. First, compliance with IC practices was based on nurses' self-reported behavior on a survey questionnaire rather than on direct observation. Previous studies comparing self-reported to observed hand hygiene practices revealed discrepancies suggesting higher adherence rates when practices were selfreported relative to those that were directly observed. ${ }^{15}$ Second, the 2 agencies surveyed were both located in the northeastern region of the United States, and it is possible that the findings observed in our sample would not generalize to HHC nurses in other areas of the country. While beyond the scope of the present study, future research should also consider how other environmental and organizational characteristics may affect IC practices, including the patient's home environment and variations in agency-level initiatives to improve compliance with IC practices. ${ }^{40}$ For instance, aspects of the home environment, including stressful and potentially hazardous working conditions, poor lighting, and extensive clutter, have been shown to increase risk for percutaneous injuries among HHC nurses. ${ }^{32}$ Furthermore, although nurses' IC practices are important to study in their own right as a prevention strategy, we recognize that the causes and spread of infections among HHC patients are likely to be complex and difficult to attribute to nurse practices. 


\section{Acknowledgments}

The authors are grateful to Gwynneth Johnson and Olivia Brisbane, students at the University Of Pennsylvania School Of Nursing, who helped gather literature and other information that assisted with the development of the survey questionnaire. We are also thankful to Tami Videon and Irene Bick for their help with survey administration.

Funding/support: The authors disclosed receipt of the following financial support for the research, authorship, and/or publication of this article: this project was supported by grant number R01HS024723 from the Agency for Healthcare Research and Quality. The content is solely the responsibility of the authors and does not necessarily represent the official views of the Agency for Healthcare Research and Quality.

\section{References}

1. MedPAC. Report to congress: medicare payment policy. 2016. Available from: http:// www.medpac.gov/docs/default-source/reports/march-2016-report-to-the-congress-medicarepayment-policy.pdf

2. Shang J, Ma C, Poghosyan L, Dowding D, Stone P. The prevalence of infections and patient risk factors in home health care: a systematic review. Am J Infect Control. 2014; 42:479-84. [PubMed: 24656786]

3. Shang J, Larson E, Liu J, Stone P. Infection in home health care: results from national Outcome and Assessment Information Set data. Am J Infect Control. 2015; 43:454-9. [PubMed: 25681302]

4. Albrich WC, Harbarth S. Health-care workers: source, vector, or victim of MRSA? Lancet Infect Dis. 2008; 8:289-301. [PubMed: 18471774]

5 . Kenneley I. Infection control in home healthcare: an exploratory study of issues for patients and providers. Home Healthc Nurse. 2012; 30:235-45. [PubMed: 22395313]

6. Bakunas-Kenneley I, Madigan EA. Infection prevention and control in home health care: the nurse's bag. Am J Infect Control. 2009; 37:687-8. [PubMed: 19556036]

7. Centers for Medicare \& Medicaid Services (CMS), HHS. Medicare and Medicaid Program: conditions of participation for Home Health Agencies. Final rule. Fed Regist. 2017; 82:4504. [PubMed: 28102984]

8. Umscheid CA, Mitchell MD, Doshi JA, Agarwal R, Williams K, Brennan PJ. Estimating the proportion of healthcare-associated infections that are reasonably preventable and the related mortality and costs. Infect Control Hosp Epidemiol. 2011; 32:101-14. [PubMed: 21460463]

9. Kenneley IL. Infection control and prevention in home healthcare: prevention activities are the key to desired patient outcomes. Home Healthc Nurse. 2007; 25:459-69. [PubMed: 17667005]

10. Kenneley IL. Infection control and prevention in home healthcare: prevention activities are the key to desired patient outcomes. Home Healthc Nurse. 2007; 25:459-67. quiz 68-9. [PubMed: 17667005]

11. Whitby M, Pessoa-Silva CL, McLaws ML, Allegranzi B, Sax H, Larson E, et al. Behavioural considerations for hand hygiene practices: the basic building blocks. J Hosp Infect. 2007; 65:1-8. [PubMed: 17145101]

12. Whitby M, McLaws ML, Ross MW. Why healthcare workers don't wash their hands: a behavioral explanation. Infect Control Hosp Epidemiol. 2006; 27:484-92. [PubMed: 16671030]

13. Valim MD, Marziale MHP, Richart-Martínez M, Sanjuan-Quiles Á. Instruments for evaluating compliance with infection control practices and factors that affect it: an integrative review. J Clin Nurs. 2014; 23:1502-19. [PubMed: 24004371]

14. Smiddy MP, O'Connell R, Creedon SA. Systematic qualitative literature review of health care workers' compliance with hand hygiene guidelines. Am J Infect Control. 2015; 43:269-74. [PubMed: 25728153]

15. Seibert DJ, Speroni KG, Oh KM, DeVoe MC, Jacobsen KH. Knowledge, perceptions, and practices of methicillin-resistant Staphylococcus aureus transmission prevention among health care workers in acute-care settings. Am J Infect Control. 2014; 42:254-9. [PubMed: 24406259]

16. Allegranzi B, Pittet D. Role of hand hygiene in healthcare-associated infection prevention. J Hosp Infect. 2009; 73:305-15. [PubMed: 19720430] 
17. McGuckin M, Waterman R, Govednik J. Hand hygiene compliance rates in the United States-a one-year multicenter collaboration using product/volume usage measurement and feedback. Am J Med Qual. 2009; 24:205-13. [PubMed: 19332864]

18. Erasmus V, Daha TJ, Brug H, Richardus JH, Behrendt MD, Vos MC, et al. Systematic review of studies on compliance with hand hygiene guidelines in hospital care. Infect Control Hosp Epidemiol. 2010; 31:283-94. [PubMed: 20088678]

19. Sax H, Perneger T, Hugonnet S, Herrault P, Chraïti M-N, Pittet D. Knowledge of standard and isolation precautions in a large teaching hospital. Infect Control Hosp Epidemiol. 2005; 26:298304. [PubMed: 15796284]

20. Efstathiou G, Papastavrou E, Raftopoulos V, Merkouris A. Factors influencing nurses' compliance with Standard Precautions in order to avoid occupational exposure to microorganisms: A focus group study. BMC Nurs. 2011; 10:1. [PubMed: 21255419]

21. Sallis JF, Owen N, Fisher E. Health behavior: theory, research, and practice. 5. San Francisco (CA): Jossey-Bass; 2015. Ecological models of health behavior; 43-64.

22. Montano DE, Kasprzyk D. Theory of reasoned action, theory of planned behavior, and the integrated behavioral model. Health behavior: theory, research and practice. 2015:95-124.

23. O'boyle CA, Henly SJ, Larson E. Understanding adherence to hand hygiene recommendations: the theory of planned behavior. Am J Infect Control. 2001; 29:352-60. [PubMed: 11743481]

24. Ajzen I. The theory of planned behaviour: reactions and reflections. Taylor \& Francis; 2011.

25. Tavolacci M-P, Ladner J, Bailly L, Merle V, Pitrou I, Czernichow P. Prevention of nosocomial infection and standard precautions: knowledge and source of information among healthcare students. Infect Control Hosp Epidemiol. 2008; 29:642-7. [PubMed: 18611166]

26. Pittet D, Allegranzi B, Boyce J. World Health Organization World Alliance for Patient Safety First Global Patient Safety Challenge Core Group of Experts. The World Health Organization guidelines on hand hygiene in health care and their consensus recommendations. Infect Control Hosp Epidemiol. 2009; 30:611-22. [PubMed: 19508124]

27. Parmeggiani C, Abbate R, Marinelli P, Angelillo IF. Healthcare workers and health care-associated infections: knowledge, attitudes, and behavior in emergency departments in Italy. BMC Infect Dis. 2010; 10:35. [PubMed: 20178573]

28. Chan R, Molassiotis A, Eunice C, Virene C, Becky H, Chit-Ying L, et al. Nurses' knowledge of and compliance with universal precautions in an acute care hospital. Int J Nurs Stud. 2002; 39:157-63. [PubMed: 11755446]

29. Gershon RR, Vlahov D, Felknor SA, Vesley D, Johnson PC, Delcios GL, et al. Compliance with universal precautions among health care workers at three regional hospitals. Am J Infect Control. 1995; 23:225-36. [PubMed: 7503434]

30. Lindberg M, Skytt B, Högman M, Carlsson M. The Multidrug-Resistant Bacteria Attitude Questionnaire: validity and understanding of responsibility for infection control in Swedish registered district, haematology and infection nurses. J Clin Nurs. 2012; 21:424-36. [PubMed: 22150887]

31. Kingston LM, Slevin BL, O'Connell NH, Dunne CP. Hand hygiene: attitudes and practices of nurses, a comparison between 2007 and 2015. Am J Infect Control. 2017

32. Gershon RR, Pearson JM, Sherman MF, Samar SM, Canton AN, Stone PW. The prevalence and risk factors for percutaneous injuries in registered nurses in the home health care sector. Am J Infect Control. 2009; 37:525-33. [PubMed: 19216006]

33. Felembam O, John WS, Shaban RZ. Hand hygiene practices of home visiting community nurses: perceptions, compliance, techniques, and contextual factors of practice using the World Health Organization's "five moments for hand hygiene". Home Healthc Nurse. 2012; 30:152-60. [PubMed: 22391658]

34. Rosati RJ, Zales M, Landers S. The benefits and challenges of the influenza vaccination among home health care workers. Home Health Care Manag Pract. 2016; 28:178-83.

35. Ajzen I, Joyce N, Sheikh S, Cote NG. Knowledge and the prediction of behavior: the role of information accuracy in the theory of planned behavior. Basic Appl Soc Psych. 2011; 33:101-17. 
36. Darawad MW, Al-Hussami M, Almhairat II, Al-Sutari M. Investigating Jordanian nurses' handwashing beliefs, attitudes, and compliance. Am J Infect Control. 2012; 40:643-7. [PubMed: 22245246]

37. Borg MA, Benbachir M, Cookson BD, Redjeb SB, Elnasser Z, Rasslan O, et al. Self-protection as a driver for hand hygiene among healthcare workers. Infect Control Hosp Epidemiol. 2009; 30:578-80. [PubMed: 19419325]

38. Saffari M, Ghanizadeh G, Fattahipour R, Khalaji K, Pakpour AH, Koenig HG. Effect of the Intelligent Health Messenger Box on health care professionals' knowledge, attitudes, and practice related to hand hygiene and hand bacteria counts. Am J Infect Control. 2016; 44:e283-5. [PubMed: 27908438]

39. Marschall P, Hübner N-O, Maletzki S, Wilke F, Dittmann K, Kramer A. Attitudes and perceptions of health care workers in Northeastern Germany about multidrug-resistant organisms. Am J Infect Control. 2016; 44:e91-4.

40. Rhinehart E, Friedman MM. Infection control in home care and hospice. Jones \& Bartlett Learning; 2005. 


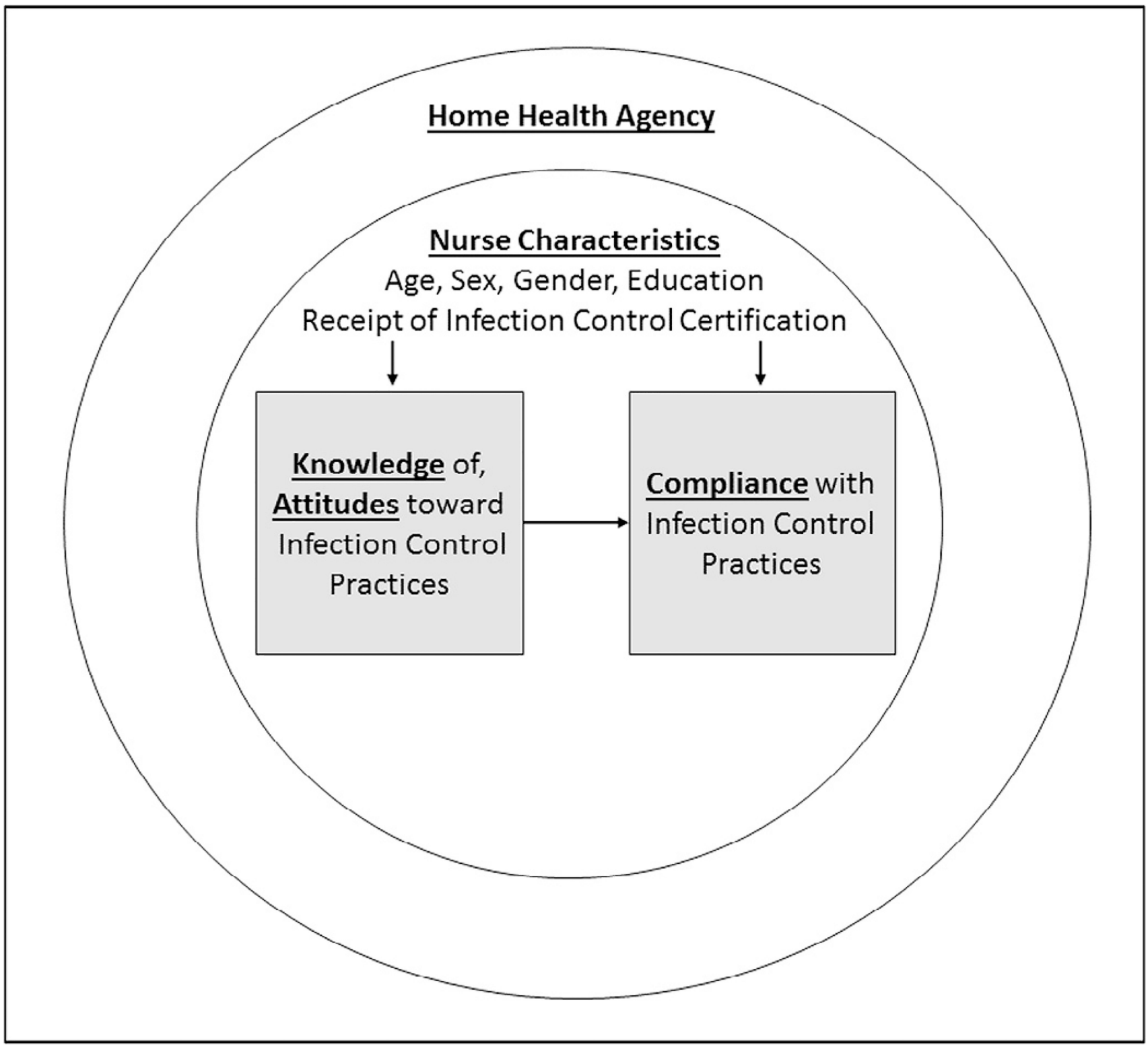

Fig 1.

Conceptual diagram 
Table 1

Demographic characteristics of nurse survey respondents for the total sample and by agency

\begin{tabular}{|c|c|c|c|}
\hline \multirow[b]{2}{*}{ Characteristic } & \multirow{2}{*}{$\frac{\text { Total Sample }(\mathbf{n}=\mathbf{3 5 9})}{\%(\mathbf{n}) \text { or M (SD) }}$} & \multirow{2}{*}{$\frac{\text { Agency } 1(n=210)}{\%(n) \text { or } M(S D)}$} & \multirow{2}{*}{$\frac{\text { Agency } 2(n=149)}{\%(n) \text { or } M(S D)}$} \\
\hline & & & \\
\hline Age & $50.0(10.5)$ & $49.6(10.6)$ & $50.5(10.3)$ \\
\hline \multicolumn{4}{|l|}{ Sex } \\
\hline Men & $8.4 \%(30)$ & $10.0 \%(21)$ & $6.0 \%(9)$ \\
\hline Women & $91.6 \%(329)$ & $90.0 \%(189)$ & $94.0 \%(140)$ \\
\hline \multicolumn{4}{|l|}{ Race/Ethnicity } \\
\hline Non-Hispanic White & $42.9 \%(154)$ & $32.4 \%(68)^{* * * *}$ & $57.7 \%(86)$ \\
\hline Non-Hispanic Black & $24.0 \%(86)$ & $26.7 \%(56)$ & $20.1 \%(30)$ \\
\hline Hispanic & $10.9 \%(39)$ & $12.9 \%(27)$ & $8.1 \%(12)$ \\
\hline Other or Mixed Race/Ethnicity & $22.3 \%(80)$ & $28.1 \%(59)$ & $14.1 \%(21)$ \\
\hline \multicolumn{4}{|l|}{ Education } \\
\hline Graduate Degree & $19.8 \%(71)$ & $26.7 \%(56)^{* * * *}$ & $10.1 \%(15)$ \\
\hline Bachelor's Degree & $51.8 \%(186)$ & $54.3 \%(114)$ & $48.3 \%(72)$ \\
\hline Associate's Degree or Some College & $28.4 \%(102)$ & $19.0 \%(40)$ & $41.6 \%(62)$ \\
\hline Years in Nursing & $21.9(11.9)$ & $22.2(11.9)$ & $21.4(11.8)$ \\
\hline Years in Home Care Nursing & $13.5(9.3)$ & $15.1(9.1)$ & $11.3(9.3)$ \\
\hline Years at Agency & $11.5(9.1)$ & $13.7(9.1)^{* * *}$ & $8.3(8.3)$ \\
\hline \multicolumn{4}{|l|}{ Position at Agency } \\
\hline Full-Time Staff Nurse & $64.9 \%(233)$ & $63.8 \%(134)$ & $66.4 \%(99)$ \\
\hline Part-Time Staff Nurse & $21.4 \%(77)$ & $20.0 \%(42)$ & $23.5 \%(35)$ \\
\hline Team Manager & $13.6 \%(49)$ & $16.2 \%(34)$ & $10.1 \%(15)$ \\
\hline \multicolumn{4}{|l|}{ Last Received Infection Control Training } \\
\hline Less than 6 Months & $39.3 \%(141)$ & $35.7 \%(75)$ & $44.3 \%(66)$ \\
\hline More than 6 Months, Less than 1 Year & $43.5 \%(156)$ & $44.8 \%(94)$ & $41.6 \%(62)$ \\
\hline More than 1 Year & $17.3 \%(62)$ & $19.5 \%(41)$ & $14.1 \%(21)$ \\
\hline \multicolumn{4}{|l|}{ Certification in Infection Control } \\
\hline Received Certification & $18.1 \%(65)$ & $26.7 \%(56)$ & $6.0 \%(9)$ \\
\hline Has Not Received Certification & $81.9 \%(294)$ & $73.3 \%(154)$ & $94.0 \%(140)$ \\
\hline \multicolumn{4}{|l|}{ Infection Control Measures } \\
\hline Proportion of Correct Knowledge Responses & $0.85(0.09)$ & $0.84(0.09)$ & $0.85(0.08)$ \\
\hline Proportion of Appropriate Attitude Responses & $0.81(0.14)$ & $0.80(0.13)$ & $0.82(0.15)$ \\
\hline Proportion of Compliant Practice Responses & $0.89(0.16)$ & $0.88(0.16)^{*}$ & $0.92(0.15)$ \\
\hline
\end{tabular}

NOTE.

Statistically significant differences between agencies are noted as $* P<.05$,

$\stackrel{* * * *}{P}<.001$.

$\mathrm{M}=$ mean; $\mathrm{SD}=$ standard deviation

Am J Infect Control. Author manuscript; available in PMC 2018 November 03. 
Table 2

Percentages of nurse survey respondents with correct infection control knowledge selections $(n=359)$

\begin{tabular}{|c|c|}
\hline & Total Sample \\
\hline Knowledge Question & $\%(\mathbf{n})$ \\
\hline Masks/goggles necessary when care unlikely to cause splashing of fluids (Disagree) & $69.6 \%(250)$ \\
\hline Standard precautions do not need to be applied to persons without infections. (Disagree) & $98.9 \%(355)$ \\
\hline \multicolumn{2}{|l|}{ Which of the following sources can harbor harmful germs on hands? } \\
\hline Jewelry worn on the hands (Yes) & $95.3 \%(342)$ \\
\hline Damaged skin (Yes) & $88.0 \%(316)$ \\
\hline Artificial fingernails (Yes) & $96.9 \%(348)$ \\
\hline Birthmarks (No) & $93.6 \%(336)$ \\
\hline \multicolumn{2}{|l|}{ Which of the following are true about multiple drug-resistant organisms? } \\
\hline Antibiotic overuse can cause the development of multiple drug-resistant organisms (Yes) & $97.8 \%(351)$ \\
\hline The organisms can be spread by direct or indirect contact (Yes) & $92.8 \%(333)$ \\
\hline Clinicians can transfer organisms through a stethoscope (Yes) & $90.8 \%(326)$ \\
\hline Gowns not required for patients with active multiple drug-resistant organisms (No) & $91.4 \%(328)$ \\
\hline Contact precautions not required for patients with active multiple drug-resistant organisms (No) & $93.9 \%(337)$ \\
\hline \multicolumn{2}{|l|}{ Which of the following are correct statements about the nursing bag? } \\
\hline There is a clean and dirty side of the nursing bag (Yes) & $93.0 \%(334)$ \\
\hline The nursing bag should be cleaned when visibly soiled (Yes) & $92.5 \%(332)$ \\
\hline Hand hygiene should be performed after touching the bag (Yes) & $70.8 \%(254)$ \\
\hline The nursing bag should have at least 2 compartments (No) & $10.3 \%(37)$ \\
\hline \multicolumn{2}{|l|}{ Which situation is appropriate to use alcohol hand rub in place of washing with soap/water? } \\
\hline After using the bathroom (No) & $77.2 \%(277)$ \\
\hline Before eating and drinking (No) & $57.1 \%(205)$ \\
\hline When hands are not visibly soiled (Yes) & $83.0 \%(298)$ \\
\hline When patient has a Clostridium difficile infection (No) & $84.1 \%(302)$ \\
\hline \multicolumn{2}{|l|}{ Standard precautions } \\
\hline Include the recommendations to protect only the patients (No) & $90.5 \%(325)$ \\
\hline Include the recommendations to protect the patients and the healthcare workers (Yes) & $92.5 \%(332)$ \\
\hline Apply to all patients (Yes) & $91.6 \%(329)$ \\
\hline Apply to only healthcare workers who have contact with bodily fluids (No) & $93.9 \%$ (337) \\
\hline
\end{tabular}

NOTE. Correct responses for each knowledge item are indicated in parentheses. 


\section{Table 3}

Percentages of nurse survey respondents who indicated appropriate attitudes toward infection control practices $(\mathrm{n}=359)$

\begin{tabular}{|c|c|}
\hline & Total Sample \\
\hline Attitude Question & $\%(\mathbf{n})$ \\
\hline Infections are a serious problem in home care & $67.7 \%(243)$ \\
\hline Patients with infections can spread their infection to healthcare workers & $81.3 \%(292)$ \\
\hline I always know when I should wear a mask & $77.4 \%(278)$ \\
\hline When I perform hand hygiene, I protect my patients from infections & $98.1 \%(352)$ \\
\hline I receive enough education to recognize infections in patients & $86.4 \%(310)$ \\
\hline Patients can develop infections from contact with people who visit or live with them & $96.4 \%(346)$ \\
\hline Influenza vaccination of patients protects them against influenza & $78.0 \%(280)$ \\
\hline Influenza vaccination of healthcare workers protects patients from influenza & $73.3 \%(263)$ \\
\hline The influenza vaccine is safe & $69.9 \%(251)$ \\
\hline Our agency makes it easy for me to stay home when I am sick & $60.4 \%(217)$ \\
\hline Our agency makes hand hygiene products easily accessible to me & $95.8 \%(344)$ \\
\hline Our agency makes masks easily accessible & $89.4 \%(321)$ \\
\hline Wearing a mask makes it hard to communicate with my patients $*$ & $44.3 \%(159)$ \\
\hline Infection prevention practices help protect me from contracting infections & $97.5 \%(350)$ \\
\hline My home care agency emphasizes the importance of infection prevention & $94.4 \%(339)$ \\
\hline If I'm busy, it's hard to prioritize infection prevention practices when caring for patients * & $86.6 \%(311)$ \\
\hline I find it difficult to insert/maintain invasive devices using aseptic technique when I'm busy * & $83.0 \%(298)$ \\
\hline
\end{tabular}

NOTE. Appropriate attitudes were reflected by responses of "strongly agree" or "agree."

reverse coded to reflect nurses who indicated "strongly disagree" or "disagree" with the listed statement. 


\section{Table 4}

Percentages of nurse survey respondents who indicated self-reported compliance with infection control practices $(n=359)$

\begin{tabular}{lr}
\hline & Total Sample \\
\cline { 2 - 2 } Compliance Item & \% (n) \\
\hline I perform hand hygiene measures before and after patient care activities & $99.4 \%(357)$ \\
I wear gloves when I anticipate exposure to bodily fluids or blood products & $100.0 \%(359)$ \\
I wash my hands or use alcohol-based handrub immediately after the removal of gloves & $95.5 \%(343)$ \\
I wear a disposable face mask whenever there is a possibility of a splash or splatter & $81.9 \%(294)$ \\
I wear a gown if soiling with blood or bodily fluids is likely & $78.8 \%(283)$ \\
I wear goggles or an eye shield when I may be exposed to bloody discharge/fluid & $69.6 \%(250)$ \\
I dispose of needles in a sharps container & $96.4 \%(346)$ \\
I dispose of all potentially contaminated materials into an impermeable bag & $91.9 \%(330)$ \\
\hline
\end{tabular}

NOTE. Compliant responses were coded as responses of "always" or "often." 
Table 5

Multivariate mixed regression model of infection control (IC) compliance among home healthcare nurses ( $\mathrm{n}=$ 359)

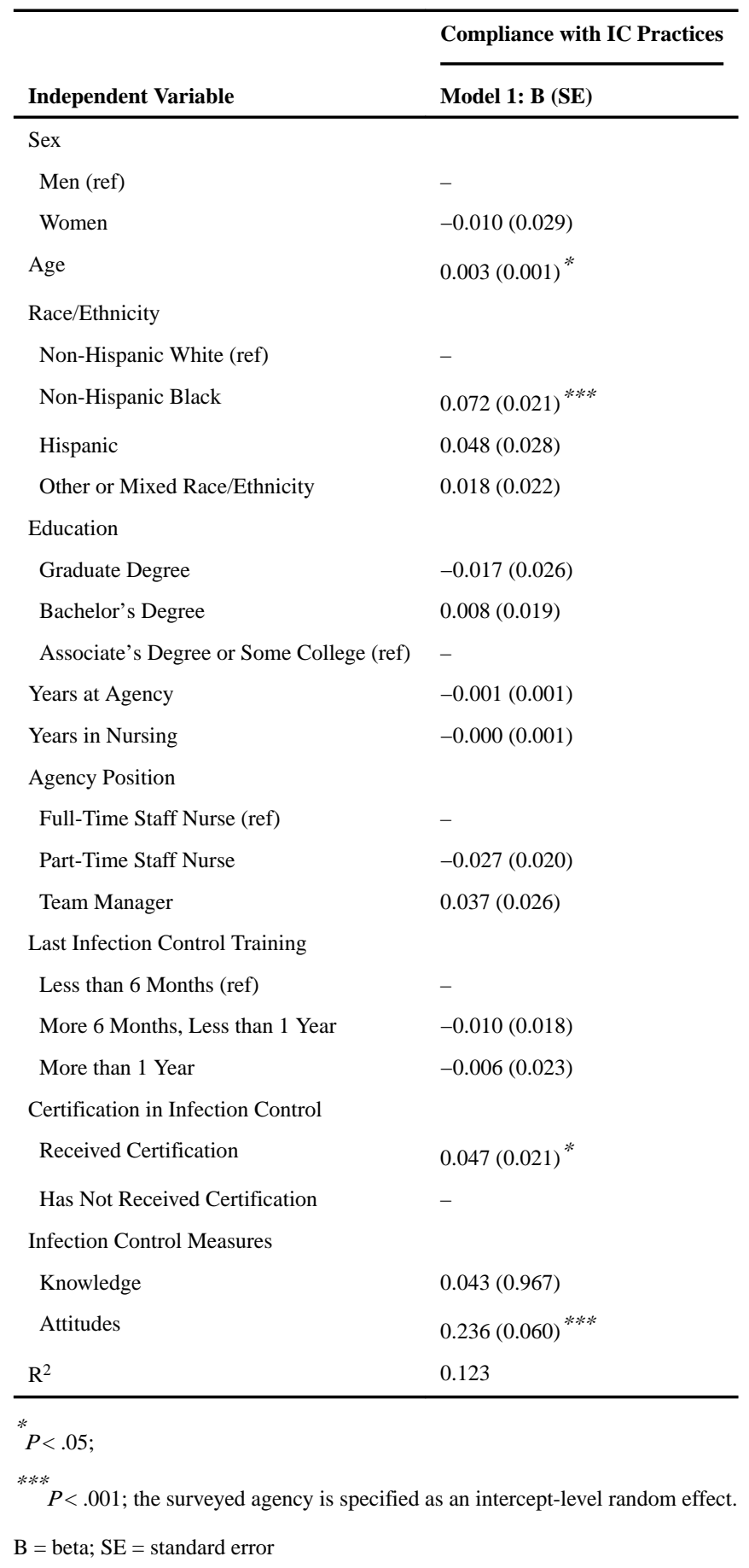

